\title{
Über das Nicht-Essen und die neue Verzichtskultur
}

\section{Eberhard Wolff}

Prof. Dr. rer. soc., Redaktor Kultur, Geschichte, Gesellschaft

«... dann sollten Sie in Zukunft glutenhaltige Produkte aus Ihrem Speiseplan streichen.» Ein Satz wie dieser gehört zum Alltag ärztlicher Empfehlung. Die Vermeidung von Lebensmitteln ist ein integraler Bestandteil von Prävention und Therapie. Etwa bei den zunehmenden Unverträglichkeiten gegenüber Nahrungsmitteln. Bei Suchtkrankheiten ist der völlige Verzicht auf das Suchtmittel die therapeutische Grundbedingung. Aber auch jenseits von Allergie und Abhängigkeit wollte und will eine Unzahl medizinischer Diäten die Gesundheit fördern, indem wahlweise Fleisch, Fett, Milch, Zucker, Salz und vieles andere mehr aus dem Speiseplan weggelassen oder die Menge reduziert wird [1]. Auf der anderen Seite kann Nahrungsenthaltung unterschiedlicher Formen als Ernährungsstörung gelten. Therapeutisches und Pathologisches kommen sich hier verdächtig nahe. Ganz zu schweigen vom Spezialfall des suizidalen Verzichts auf Essen oder Trinken: dem «Sterbefasten» [2].

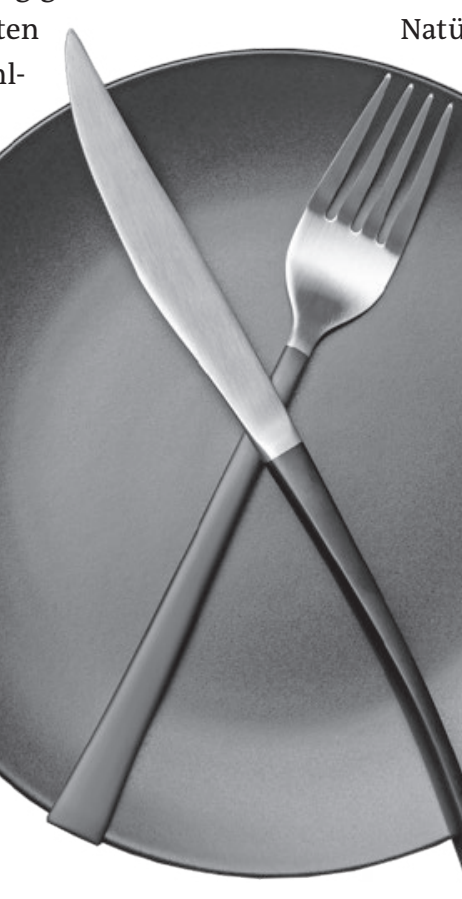

\section{Der Essensverzicht ist nah am Religiösen}

Das gewollte, bewusste Nicht-Essen hat viele medizinische Gesichter. Und noch mehr nichtmedizinische. Der zunehmende Verzicht auf den Konsum von Fleisch, «veggie», "vegan» oder jetzt "plant based», ist ein plastisches Beispiel für die kaum entwirrbare, ja gewollte Verwobenheit seiner unterschiedlichen Begründungen. Neben den gesundheitlichen Argumenten geben vegetarisch orientierte Menschen unter anderem politische, moralische, ökonomische, ästhetische und selbsterzieherische Motive für ihre Wahl an. Nicht zu vergessen, der Essensverzicht ist nah am Religiösen angesiedelt, was man besonders in der Vor-Osterzeit bemerkt, wenn das Fleischfasten neuerdings auf mal mehr, mal weniger säkulare Varianten wie das Süssigkeiten-Fasten oder die AlkoholAbstinenz ausgeweitet wird. Selbst beim Hungerstreik verbindet die bewusste Selbstgefährdung Gesundheit und Politik.

\section{Nahrung schafft Identiät}

Natürlich hat das Nicht-Essen eine lange Geschichte. Religiöse Nahrungsverbote sind so alt wie die Religionen selbst.

In der Frühen Neuzeit etwa gab es "Fastenwunder», die jahrelange Nahrungsenthaltung «in frommer imitatio und betrügerischer simulatio» zumindest vorgaben [3]. Aber erst im Laufe der hochindustriellen Moderne, seit anderthalb Jahrhunderten, wurde der säkulare Nahrungsverzicht $\mathrm{zu}$ einer verbreiteten Norm grosser Teile der Bevölkerung. Davon handelt ein Sammelband, dem viele Beispiele und Anregungen für diesen Artikel entnommen sind [4]. Abnehmkuren und Vegetarismus wurden in etwa gleichzeitig in den neuen Mittelschichten populär. Das war zu einer Zeit, als die Lebensmittelversorgung immer besser gesichert werden konnte und die Menschen ihr Leben und Essen freier gestalten konnten. Man setzte sich nun nicht mehr durch Völlerei und Körperfülle von der Masse ab, sondern dadurch, weniger und Ausgewählteres zu sich zu nehmen. Im Essen wurde die eigene Identität behauptet: Deutsche Kolonisten in Afrika etwa weigerten sich, lokale Produkte zu essen, um sich von der indigenen Bevölkerung abzusetzen [4, 237ff.]. Und ganz persönlich: Mein eigenes Verschmähen von deutschem Käse und Schweizer Bier erinnert mich an meine hybride Identität. 


\section{Das (Nicht-)Essen als Spiegel der Wertesysteme}

Das «richtige Essen" ist heute ein «neues politisches Leitnarrativ» [4, S. 41], das sich dominant im Nicht-Essen zeigt. Fast alles, was jenseits von Salat und Gemüse aus dem eigenen Kochtopf sowie Hahnenwasser angesiedelt ist, kann unter ein Verdikt fallen. Bestimmte Hersteller werden gemieden, Produktionsländer und -technologien boykottiert. Nicht-Essen ist spätestens heute zu einem festen Teil des Lebensstils geworden, in dem sich die Wertesysteme der Menschen spiegeln. Sei das nun, um eine gute Figur zu haben oder den Regenwald retten zu wollen. Abstrakt gesprochen: Nicht-Essen kommt mehr als nur dem ethisierenden Trend zu Nachhaltigkeit und Ökologie entgegen, sondern auch dem, was mit dem unglücklichen Begriff der «Selbstoptimierung» bezeichnet wird: Der intensiven

\section{Das Fundament legten vor mehr als hundert Jahren die Reformköstler durch ihre exzessive Beschäftigung mit dem (eigenen) Leib.}

Arbeit an sich selbst und der eigenen Erfolgs- und Leis tungsfähigkeit. Ein Fundament hierfür legten bereits vor mehr als einhundert Jahren die vielfältigen Reformköstler durch ihre exzessive Beschäftigung mit dem (eigenen) Leib - etwa im Zürcher Rohkost-Sanatorium des Müesli-Erfinders Max Bircher-Benner [5]. Überhaupt war die ganze Reformbewegung auch ein grosses Fest der Vermeidung: Kein Fleisch, kein Gekochtes, kein Alkohol, keine Kleider, keine Pharmazeutika.

\section{"Empowerment» oder Zwangsjacke der Selbstkontrolle?}

Dabei ist das Nicht-Essen heute auch nur Teil einer sich breit machenden kollektiven Verzichts-Kultur, die nicht nur den Cervelat, sondern aus ähnlich vielfältigen Gründen auch die Flugreise, die Autofahrt zur Arbeit und die Plastiktüte aus dem Alltag streichen will.
Je mehr es sich ausbreitet, um so kontroverser wird das Nicht-Essen eingeschätzt. Verfechter sehen darin einen Trend, die Verantwortung für die eigene Gesundheit oder die Umwelt selbst zu übernehmen, sich zu "empowern". Die anderen beurteilen den um sich greifenden Verzicht als eine gesellschaftliche Verschiebung von

\section{Manche beurteilen den um sich greifenden}

\section{Verzicht als gesellschaftliche Verschiebung der} Verantwortung auf das Individuum.

Verantwortung auf das Individuum und eine immer weiter gehende Verstrickung in einem Geflecht von Regel- und Normsystemen, eine sich immer enger um uns schliessende Zwangsjacke moralisierter Selbstund Fremdkontrolle.

Ein ganz aktuelles Beispiel: Eine Veranstaltung der Paulus-Akademie diesen November baut auf dem Untergangs-Szenario des «Wie retten wir unseren Planeten?» auf. Mit dem Titel «Verzicht oder Verbot?» fragt der Diskussionsabend gar nicht mehr, ob wir verzichten wollen, sondern nur noch, ob wir auf der Basis von Selbst- oder Fremdkontrolle verzichten sollen.

Der ärztliche Umgang mit dem Nicht-Essen, seien es medizinische Unverträglichkeiten, gezielte Diäten oder Essstörungen, findet in diesem Umfeld und eng verwoben mit ihm statt.

Bildnachweis

(c) Chernetskaya | Dreamstime.com, Symbolbild

Literatur

1 In der SÄZ hierzu z.B.: Stalder H. Richtig essen (und trinken). Schweiz Ärzteztg 2017;98(47):1594. Taverna E. Vor-Sorge. Schweiz Ärzteztg 2017;98(07):232. Ders.: Fressmythen. Schweiz Ärzteztg 2015;96(25):940.

2 Fehn S, Fringer A. Notwendigkeit, Sterbefasten differenzierter zu betrachten. Schweiz Ärzteztg 2017;98(36):1161-3.

3 Pulz W. Nüchternes Kalkül, verzehrende Leidenschaft. Nahrungsabstinenz im 16. Jahrhundert. Göttingen: Vandenhoeck \& Rup recht; 2007. Zu späteren "Hungerkünstlern» s. Danieli E. Fasten als Freakshow. Schweiz Ärzteztg 2004:85(04): 183.

4 Aselmeyer N, Settele V (eds.). Geschichte des Nicht-Essens. Verzicht, Vermeidung und Verweigerung in der Moderne. Berlin: de Gruyter; 2019.

5 Wolff E (ed.): Lebendige Kraft. Max Bircher-Benner und sein Sanatorium im historischen Kontext. Baden: hier+jetzt;2010. 